\title{
LAN TIMES GUIDE TO SECURITY AND DATA INTEGRITY
}

\author{
by Marc Farley, Tom Stearns, and Jeffrey Hsu \\ Mc Graw-Hill, 1996. ISBN: 0-7-882166-6.
}

$\mathrm{T}$ The old Chinese proverb, "May you live in interesting times," applies as much today as it ever has, especially in the word of computer networking. The rapid growth of the Internet in the last 18 months has left many networking professionals wondering what will come next. Foremost among their concerns are questions about data protection. While many people view the Internet enthusiastically as the next great computing renaissance, many of the people who responsibly manage networks that attach to the Internet fear the unknown risks to their data. But the Internet is not the only cause for alarm. Indeed, most of the changes on networks today are internally generated. Companies are increasingly dependent on their LANs to support important business functions, resulting in an increase in LAN-resident data and a healthy concern over its safety. As the amount of data grows administrators have to look for new technologies and techniques that provide the protection they need.

This book is intended to help LAN administrators understand the issues and technology of data protection in this changing world. It uses a multidisciplinary approach to give the reader a broad perspective. Therefore, a wide range of topics are presented, including backup and recovery, archiving, hierarchical storage management (HSM), redundant systems, system security, user security management and policies, authentication, encryption, viruses, and disaster recovery planning.

The first two chapters are intended to familiarize readers with the status of network data protection today, including an examination of the threats that could cause data to be lost or stolen. Chapter 3 is an in-depth analysis of LAN backup, the foundation of any data protection scheme. It includes a discussion of the problems that cause backup systems to fail and the various technologies that can be employed to solve the problems. The fourth chapter looks at ways to manage data growth more effectively through archiving and HSM techniques. In Chapter 5, several ways are examined to implement redundancy to protect LAN systems and data. 
Chapter 6 switches the focus to database systems, particularly the problems of backing up database systems on LANs. The issues of database protection are continued in the next chapter, which discusses the issues of security problems associated with database systems.

The topic of security on LANs is continued in Chapter 8 through 11. General system security, network security, advanced technologies for authentication and encryption on networks, viruses and virus protection are described in detail. The last two chapters of the book deal with future considerations. Chapter 12 looks at disaster recovery planning, and how the reader might best prepare to avoid the businessruining calamity that could happen someday. Finally, chapter 13 examines developing trends in computing technology today and attempts to predict where some potentially dangerous exposures lie for data in the future.

\section{About the authors:}

Marc Farley has a wealth of experience helping end users select and implement backup and recovery systems for their LANs. He also worked with storage experts throughout the computer industry, assisting them in the development and delivery of LAN backup and recovery solutions to their customers. Tom Stearns is a computer consultant specializing in Xbase work. He is also the co-author of Visual FoxPro Programming Basics. Jennifer Hsu is an experience author, consultant, and journalist specializing in the area of computers and scientific technologies. He has over a decade of teaching and training experience and is currently a Professor of Information Systems at Montclair State University. 